\title{
Abilities Towards Improved Accuracy in EPMA
}

\author{
Frank Eggert
}

Ametek / EDAX LLC, Mahwah, New Jersey, United States

There is no doubt that ZAF- and $\phi(\rho z)$ - models can be morphed into each other. ZAF was the first developed matrix correction, and it calculates the generation of X-rays and the absorption processes completely with separated model parts (the $\mathrm{Z}$ and the $\mathrm{A}$ in ZAF). For this reason, it classically required the A-correction to have a depth-distribution formula, the $\phi(\rho z)$, which is possible to integrate analytical over the depth with the aim to get a formula to be used in computer codes for calculation. $\phi(\rho z)$ models were developed first to overcome problems for low-energy X-rays, then to improve the analyses of thin layers. The depth distributions were measured, or Monte-Carlo method-based simulated, actually a blend of both (good overview is [1]). Because the measured/simulated and best match parameterized curves are not possible to integrate analytically, the integration is performed numerically in the computer program. With numerical integration over all depths into the specimen, with each step the generated X-rays are already corrected in regards the absorption on their path to the detector. Therefore, the $\phi(\rho z)$-method performs the ZA of ZAF in one step. On the other hand, there is no reason that the $\phi(\rho z)$-model used curves cannot also be used for ZAF, but then it is required to solve the separate A-correction with numerical integration.

The core advantage of the $\phi(\rho z)$-method is that its basic 'genes' are based on an empirically measured database. This is not in the same level with conventional ZAF, while one needs to admit that the ZAF is also using data and parameters, with the values were tweaked by measurements, and was supported in the early years also by MC-simulation.

The question is what is the best possible method to improve a ZAF model also by empirical measurement supported databases? The separation of generation and absorption is already an advantage for this purpose. We have introduced so called SCC-factors (Standards Customized Coefficients) which are one value for each element-line series (element-shell). It is useful to improve the model equations in regards of generation of X-rays which can be interpreted as an adjustment of excitation cross-sections. And because there are different datasets possible for different primarily electron energies, one can correct the algorithm also for different primarily electron energies (e.g. to adjust for critical over-voltages) [2]. As it was already pointed out [1], the MACs are the most crucial part in all absorption considerations. Therefore, the model and used MACs hang close to each other, one cannot simply replace the MACs (even if they might be better) and then still expect Quant accuracy over all specimen compositions. The EDAX eZAF correction is using [3]. The database was developed for XRF, so it is reasonable that improvements are likely required for low-Z and low X-ray energies. It is the point that the eZAF gets improved by measurements with a MAC-Correction factors (MACC) database to adjust results for non-linear runaways which occur for highabsorption cases.

However, the best would be to avoid any correction of raw measured data, except for the needed window and detector efficiencies, if required. The raw measured data are normally net-counts (CPS), ratios of measured net-counts (k-ratios), or net-counts divided by measured bremsstrahlung counts of same energy (P/B). It was already discussed that the $\mathrm{P} / \mathrm{B}$ method has the advantage of much less model-correction requirements, especially since the absorption is typically one order of magnitude less [4]. The k-ratios are always used in standards comparison, for each element line of unknown sample, and it is required to have at least one measured standard. If an unknown compound measurement is only partially supported by 
standards, then the remained are calculated standardless (net-counts of the pure element standard in kratios are then calculated, not measured). Any deviation of unknown specimen concentrations from the used standard is moving away from the ideal k-ratio value of 1.000. The deviations produce the requirement to apply corrections (as well as the non-standard supported elements need the complete standardless model). The model influences rise with growing deviations between standard and unknown composition. With this also the uncertainty of model contributes more to result uncertainties. The SCC and MACC empirical adjustment also affect in most cases the Full Standards Quant (FSQ) method results. The ideal case goal is to select standards reasonable to have no corrections (means no model influence).

There are different possible strategies. One is to measure simple standards which are collected in own lab and then to utilize stored standards data which are closer to the unknown sample compositions, provided by a huge database [5]. The elsewhere measured standards library data are required to relate with own instrument measurements. We have tested a similar strategy to import standards which were measured elsewhere by retracing it to the current instrument with only a pure element reference measurement. The "reference" is used to tag the different solid-angle and beam current base setup. The database standards need always a tag with the 'reference' and the reference measurement is then required to perform also at SEM/EDS user site. The standard can be even measured with another EDS model or windows-type, the proper consideration of different efficiency is then required.

In the ideal case with a huge database there will be a standard available for any potential unknown specimen. But in realistic terms there will still be gaps. And the question is how to select the best matching standard(s) for the stated goal to keep k-ratios close to 1.000 for the aim to get the applied corrections as tiny as possible. A flexible standards Quant algorithm was tested which considers the standards by using an iterative method with selecting the best matching standard(s) during evaluation.

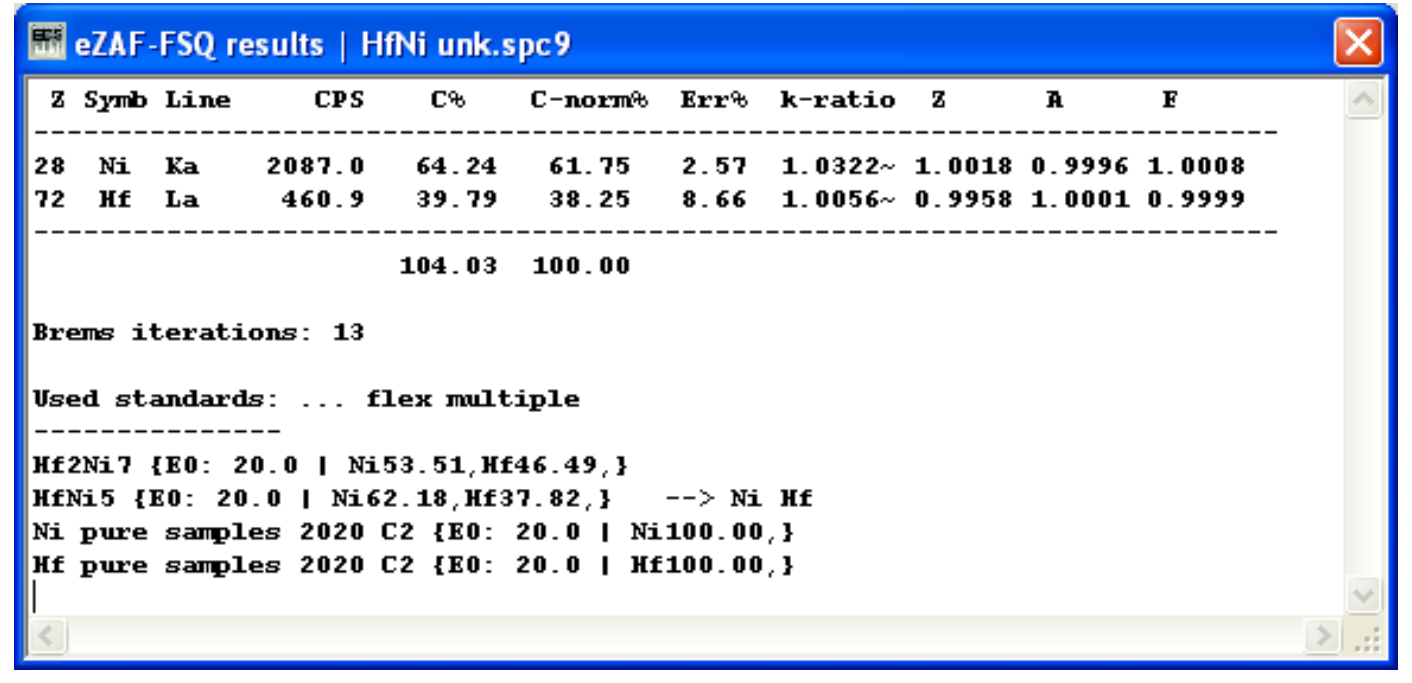

Figure 1. Three standards are provided usable for Hf-L. The one most close was automatically selected and effectively there is then no remained model correction with ZAF and with this no uncertainty influence by model. 


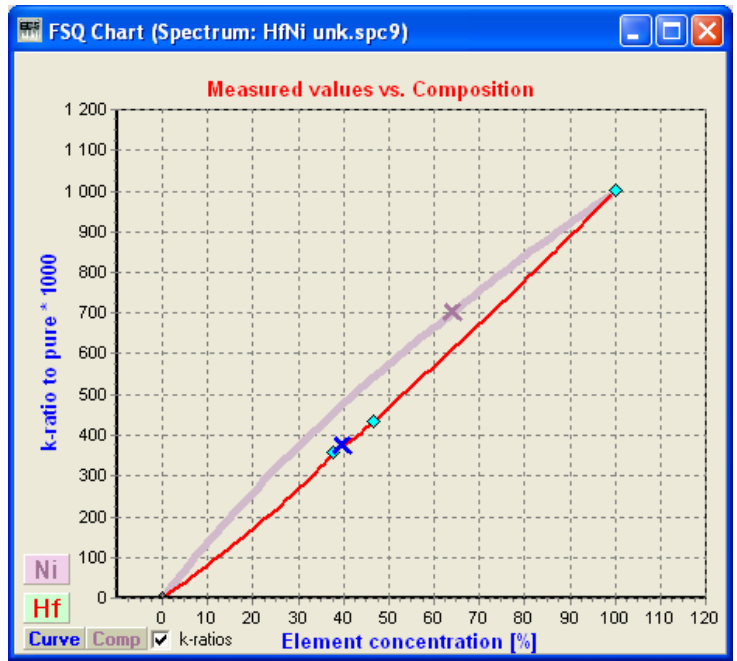

Figure 2. The red line is calibrated quantitative curve for Hf with the use of 3 standards.

\section{References}

[1] X Llovet at al, Progress in Materials Science 116 (2021) 100673

[2] J Rafaelsen, S Mu, Microsc. Microanal. 26 Suppl 2 (2020) 2174

[3] W T Elam, B D Ravel, J R Sieber Radiation Physics and Chemistry 63/2 (2002) 121

[4] F Eggert, Microscopy Today 28/2 (2020) 34

[5] N Ritchie at al, Microsc. Microanal. 26 Suppl 2 (2020) 1774 